\title{
Immunoinformatics analysis and antibody epitope comparison of Newcastle disease virus classical vaccines with a virus involved in the fifth NDV panzootic
}

Seyed-Elias Tabatabaeizadeh ( $\nabla$ e.tabatabaee@rvsri.ac.ir)

Razi Vaccine and Serum Research Institute https://orcid.org/0000-0002-5914-467X

\section{Research Article}

Keywords: Newcastle disease virus, vaccine strain, conformational epitope, linear B-cell epitopes, immunoinformatics

Posted Date: April 26th, 2021

DOl: https://doi.org/10.21203/rs.3.rs-456948/v1

License: (c) (i) This work is licensed under a Creative Commons Attribution 4.0 International License. Read Full License 


\section{Abstract}

Newcastle disease virus (NDV) has negatively affected the poultry industry worldwide. Given that the antigenic similarity of a vaccine strain to a field virus is effective in protection, an immunoinformatics study was performed to examine the similarity between antibody epitopes of classical vaccines and a sub-genotype VII.2 NDV (VII.2 NDV). Considering the role of fusion (F) and hemagglutinin-neuraminidase $(\mathrm{HN})$ proteins in the induction of neutralizing antibodies, the 3D structure of $\mathrm{HN}$ and $\mathrm{F}$ proteins of the VII.2 NDV and nine vaccine strains were predicted, refined, and validated. Using these structures, linear and conformational antibody epitopes were mapped. Epitope analysis showed distinct results from the evolutionary distance and protein identity analysis and it was found that the range of difference in the number of identical epitopes in relation to $\mathrm{F}$ is wider than $\mathrm{HN}$ protein. LaSota and $\mathrm{B} 1$ vaccine strains showed the least epitope identity to the VII.2 NDV. The V4 and I-2 vaccine strains showed the highest epitope identity with the VII.2 NDV especially in F protein which is important in virus cell-to-cell transmission. In conclusion, excellence of the LaSota vaccine under field condition shows that protection is not just about epitope similarities and especially in the case of live vaccines, the vaccine-induced damage, replicative capacity and tropism of the vaccine strain are important. The prediction of this study may be useful for inactivated vaccines in which the amount of antigen is all that matters.

\section{Introduction}

Newcastle disease virus (NDV), also known as avian paramyxovirus 1 (APMV-1), is a virus of genus avian orthoavulavirus 1 (AOAV-1) with worldwide distribution (ICTV 2019; Suarez et al. 2020). NDV can infect more than 200 species of birds, however, the Newcastle disease (ND) severity is dependent on factors like virus strain, host, the presence of other organisms, and environmental conditions (Suarez et al. 2020). Because of negative effects on the world poultry industry, the World Organization for Animal Health listed ND as a notifiable terrestrial animal disease. NDV genome is a negative sense, single-stranded, nonsegmented RNA with a size of about $15.2 \mathrm{~kb}$ that encodes 6 structural proteins: nucleocapsid protein $(\mathrm{N})$, phosphoprotein $(P)$, matrix $(M)$, fusion $(F)$, hemagglutinin-neuraminidase $(H N)$, and large polymerase $(L)$ (Suarez et al. 2020).

$\mathrm{HN}$ and $\mathrm{F}$ proteins are located at the outer surface of the enveloped virus and are important in immunoprotection (Merz et al. 1980; Boursnell et al. 1990; Naohiro et al. 1994; Reynolds and Maraqa 2000). HN protein is involved in virus binding to sialic acid receptors of cells, neuraminidase activity, and through interaction with $\mathrm{F}$ protein, mediates fusion of the viral envelope with the cell membrane (Suarez et al. 2020). According to the virulence, isolates of NDV are categorized into three main pathotypes: lentogenic (low virulence), mesogenic (moderate virulence), and velogenic (high virulence) (Suarez et al. 2020). Avirulent and lentogenic strains have been used extensively as live vaccines (Dimitrov et al. 2017). Although all isolates of NDV are grouped serologically into a single serotype (Suarez et al. 2020), but vaccine protection is not always optimal. This may be due to poor vaccination practices or different antigenic properties of the circulating virulent NDVs and the vaccine strains (Miller et al. 2007; Dortmans et al. 2012; Liu et al. 2017). Considering the important role of F and HN proteins in virus tropism, 
pathogenicity, and virulence (Römer-Oberdörfer et al. 2003; Römer-Oberdörfer et al. 2006; Khattar et al. 2009), it was proved that some antibodies against these glycoproteins can neutralize NDV and are important in the induction of protective immunity (Lee et al. 2008; Kim et al. 2013; Liu et al. 2017).

Different strains of classical NDV vaccines are now available, and in addition to their high safety profile, have been protective (Dortmans et al. 2012; Dimitrov et al. 2017). On the other hand, the antigenic similarity between a vaccine strain and the field virus may improve vaccination-induced protection in terms of infectivity and virus shedding (Miller et al. 2009; Miller et al. 2013; Yang et al. 2017; Shahar et al. 2018).

Viruses of sub-genotype VII.2 are responsible for the fifth NDV panzootic that affected the Middle East, Asia, Europe, and Africa (Fuller et al. 2017; Abolnik et al. 2018; Ghalyanchilangeroudi et al. 2018; Dimitrov et al. 2019). This study was performed to identify antibody epitopes of nine classical vaccine strains. These epitopes were compared with their analogous ones identified from a virulent NDV of sub-genotype VII.2.

\section{Materials And Methods}

\subsection{Collection of $\mathrm{HN}$ and $F$ proteins of vaccine strains and sub-genotype VII.2 NDV}

The full-length amino acid sequences of $\mathrm{HN}$ and $\mathrm{F}$ proteins of nine live attenuated NDV vaccine strains, including B1 (AF309418.1), I-2 (AY935499.2), PHY-LMV42 (DQ097394.1), R2B (JX316216.1), Ulster (AY562991.1), V4 (JX524203.1), VG/GA (KC906188.1), LaSota (AF077761.1), and F (KC987036.1) were retrieved from GenBank. The HN protein of PHY-LMV42, Ulster, V4, and VG/GA strains is longer than the one of the other NDV vaccine strains. However, for $\mathrm{HN}$ protein activation, this $\mathrm{C}$-terminal extension is removed by proteolytic cleavage (Gorman et al. 1988). Accordingly, $47 \mathrm{C}$-terminal amino acids were removed from the HN protein of these strains before bioinformatics analysis. The full-length amino acid sequence of $\mathrm{HN}$ and $\mathrm{F}$ proteins of a virulent NDV with a characterized genome (MH614933.1) (Ababneh et al. 2018) used as the representative of sub-genotype VII.2 NDV (hereafter, referred to as VII.2 NDV).

\subsection{Estimates of evolutionary distance and identity analysis of antigens}

The amino acid sequences of $\mathrm{HN}$ and $\mathrm{F}$ proteins were aligned using the MUSCLE algorithm and the evolutionary distances were calculated in MEGA X (Kumar et al. 2018). The identity percentage between the $\mathrm{HN}$ and $\mathrm{F}$ protein sequences of the VII.2 NDV and the vaccine strains was calculated using the NCBI BLAST (https://blast.ncbi.nlm.nih.gov/Blast.cgi).

\subsection{D structure modeling of HN and F proteins of the vaccine strains and the VII.2 NDV}

Since most antibodies identify conformational epitopes (Barlow et al. 1986; Van Regenmortel 1996), the $3 D$ structures of $\mathrm{HN}$ and $\mathrm{F}$ proteins were constructed for antibody epitope prediction. MODELLER program version 9.23 was used for the model building of HN proteins (Sali and Blundell 1993). MODELLER implements the "modeling by satisfaction of spatial restraints". For comparative modeling of the vaccine 
strains and the VII.2 NDV, X-ray diffraction derived HN structure (PDB code 1E8T) was used as a template (Crennell et al. 2000). Amino acid residues 124-570 of HN protein were used for comparative modeling. Xray diffraction derived $F$ structure in the prefusion state (PDB code $1 \mathrm{G} 5 \mathrm{G}$ ) was used as a template (Chen et al. 2001). Amino acid residues 106-170 of $\mathrm{F}$ protein are not modeled in $1 \mathrm{G} 5 \mathrm{G}$ structure, and it caused that MODELLER program output has a different structure at the stalk region compared to $1 \mathrm{G} 5 \mathrm{G}$.

Therefore, I-TASSER (Iterative Threading ASSEmbly Refinement) on-line server, with specifying 1G5G as a template, used for homology modeling of $F$ proteins (Roy et al. 2010). The I-TASSER server has been ranked as the top server for protein structure prediction in recent community-wide CASP experiments (Moult et al. 2018). Amino acid residues 33-454 of F protein were used for comparative modeling.

\subsection{D structure refinement and validation}

The 3D structures modeled for the $\mathrm{HN}$ and $\mathrm{F}$ proteins were refined using the GalaxyRefine server (http://galaxy.seoklab.org/cgi-bin/submit.cgi?type=REFINE). The GalaxyRefine server has been tested successfully in community-wide CASP10 experiments. This method rebuilds and then repacks side chains, and causes a relaxation of overall protein structure using molecular dynamics simulation. This leads to an improvement in the quality of both global and local structures (Heo et al. 2013). After refinement of modeled proteins, the GalaxyRefine server provided data about MolProbity, Clash score, Poor rotamers, and Ramachandran plot analysis which were used for validation of the refined protein structures. ProSA-web (https://prosa.services.came.sbg.ac.at/prosa.php) was also used to validate the refined protein structures. Using ProSA the overall quality of proteins was evaluated and compared in the context of the previously known protein structures (Wiederstein and Sippl 2007). Structure files in PDB format for the modeled $\mathrm{HN}$ and $\mathrm{F}$ proteins are included in supplementary information files.

\subsection{The prediction of antibody epitopes}

Discontinuous or conformational epitopes are composed of amino acids that coming together in the surface of an antigen structure. Conformational epitopes make up more than $90 \%$ of antibody epitopes in an antigen (Barlow et al. 1986; Van Regenmortel 1996). ElliPro is a web-tool that identifies linear and conformational antibody epitopes using an antigen 3D structure as an input. By giving an AUC value of 0.732, ElliPro performed the best in antibody epitope prediction compared to the other structure-based methods (Ponomarenko et al. 2008). ElliPro predicts antibody epitopes by implementing algorithms that lead to an approximation of protein shape as an ellipsoid, calculation of the residue protrusion index (PI), and using PI values for neighboring residues clustering. Regarding the epitope prediction parameters, the minimum score and the maximum distance were specified at 0.5 and 5 angstroms, respectively. The $F$ and $\mathrm{HN}$ proteins form trimers and tetramers on the virion, respectively. After epitope mapping, epitopes that were located at the interface of each monomer were omitted from the study.

\subsection{Estimating the epitope identity between vaccine strains and the sub-genotype VII.2 NDV}

To find the best vaccine strain, it was necessary to make an appropriate comparison between the identity level of analogous epitopes of the VII.2 NDV and each vaccine strain. To calculate the identity percentage 
between two analogous epitopes, it was considered that the presence of a different amino acid in a vaccine strain epitope compared to its absence possibly has a greater effect on reducing antibody binding. The identity is defined in equation 1, where aa stands for amino acids and ae for the analogous epitope.

(1)

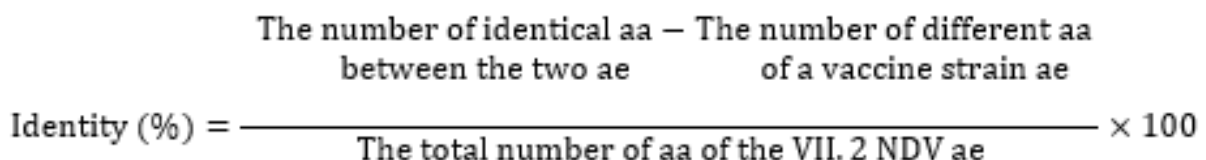

\section{Results}

\subsection{The evolutionary distance and identity between HN and F proteins of the sub-genotype VII.2 NDV and vaccine strains}

To have a preliminary comparison between the HN and F proteins of the VII.2 NDV and vaccine strains regarding the number of amino acid substitutions per site, the evolutionary distance was calculated (Table 1). The number of amino acid substitutions per site between sequences is higher for HN $(0.125$ average) than $\mathrm{F}$ (0.105 average) protein. HN protein of the VII.2 NDV has the highest and the lowest evolutionary distances with F and Ulster strains, respectively. Regarding F protein, V4 and VG/GA strains have the lowest and B1 strain has the highest evolutionary distances. Protein sequence identity analysis shows that the HN protein of the VII.2 NDV has the highest and the lowest identity with Ulster and F strains, respectively, and the F protein has the highest identity with V4 and VG/GA strains and the lowest identity with B1 strain (Table 1).

The number of amino acid substitutions per site and identity for HN protein ranged from 0.109 to 0.147 (0.038 difference) and 86.64 to $89.98 \%$ (3.34 difference), respectively. The number of amino acid substitutions per site and identity for $F$ protein ranged from 0.089 to 0.125 (0.036 difference) and 88.41 to $91.67 \%$ (3.26 difference), respectively.

\subsection{D structure modeling}

For HN protein structure prediction, homology modeling was performed by MODELLER program version 9.23 employing basic modeling. Using the command model-single.py, the DOPE score was calculated for the predicted structures. Out of the five HN models predicted for each virus, the model with the lowest DOPE score was selected for further refinement. For F protein structure prediction, five structures were modeled for each virus using the I-TASSER server based on 10 threading templates. The threading alignments had a normalized Z-score from 2 to 10 which means a good alignment. Out of the 5 modeled structures predicted for each F protein, the best model with the highest C-score was selected and used for further refinement. 


\subsection{The refinement and validation of 3D structures}

The GalaxyRefine server was employed to refine the selected models of $\mathrm{HN}$ and $\mathrm{F}$ proteins. It produced five refined protein structures for each modeled protein. According to the calculated parameters, the bestrefined models were selected (Table 2). MolProbity scores of the modeled proteins were in the range of 1 to 2 which is typical for experimental structures. ProSA-web was also used to obtain the overall quality score for the modeled proteins. The Z-score of the modeled proteins was within the range of scores typically found for native proteins of similar size.

\subsection{Prediction of linear and conformational antibody epitopes}

ElliPro was used for the antibody epitope prediction employing the modeled $\mathrm{HN}$ and $\mathrm{F}$ proteins. The position of the predicted antibody epitopes in the 3D structure of $\mathrm{HN}$ and F proteins of the VII.2 NDV are shown in figures 1 and 2, respectively.

Linear and conformational antibody epitopes of HN proteins are shown in tables 3 and 4, respectively.

Linear and conformational antibody epitopes of $F$ proteins are depicted in tables 5 and 6 , respectively.

In the case of both $\mathrm{HN}$ and $\mathrm{F}$ proteins, some epitopes detected in silico incorporated amino acids that experimental findings have been previously shown that are involved in the construction of neutralizing epitopes (Tables 3-6) (Chambers et al. 1988; Toyoda et al. 1988; Neyt et al. 1989; Yusoff et al. 1989; lorio et al. 1991; lorio et al. 1992).

\subsection{Identity analysis between analogous epitopes of the vaccine strains and the VII.2 NDV}

The identity percentage between analogous epitopes was calculated according to equation 1 . The number of analogous epitopes between the VII.2 NDV and the vaccine strains has been shown in table 7 for each epitope identity percentage range. Different percentages of identity were observed for both proteins in the case of linear and conformational epitopes. F protein identical linear and conformational epitopes are ranged from 1 to 5 , and $\mathrm{HN}$ protein identical linear and conformational epitopes are respectively ranged from 0 to 2 and 2 to 3 . Totally, the number of identical epitopes of $F$ and $\mathrm{HN}$ proteins is respectively ranged from 3 to 10 and 2 to 5 (Table 7).

The order of the vaccine strains in relation to the number of $F$ protein identical linear and conformational epitopes to the VII.2 NDV is as follows: $(\mathrm{V} 4=10)>(F=7 ; \mathrm{R} 2 \mathrm{~B}=7 ; \mathrm{I}-2=7)>(\mathrm{VG} / \mathrm{GA}=6)>(\mathrm{Ulster}=5)>$ $($ LaSota $=4)>($ B1 =3; PHY.LMV.42=3).

The order of the vaccine strains in relation to the number of $\mathrm{HN}$ protein identical linear and conformational epitopes to the VII.2 NDV is as follows: (Ulster=5) $>(I-2=4 ; \mathrm{V} 4=4 ; \mathrm{VG} / \mathrm{GA}=4)>($ LaSota $=3$; PHY.LMV.42=3; R2B=3) > (B1=2; F=2).

The order of the vaccine strains in relation to the number of identical linear and conformational epitopes of $\mathrm{F}$ and HN proteins to the VII. $2 \mathrm{NDV}$ is as follows: $(\mathrm{V} 4=14)>(I-2=11)>($ Ulster=10; VG/GA=10; R2B=10) > 
$(\mathrm{F}=9)>($ LaSota $=7)>($ PHY.LMV.42=6) $>(\mathrm{B} 1=5)$.

\section{Discussion}

This study was conducted to answer the question of how much the antigenic similarity of classical NDV vaccine strains with circulating virulent NDV strains can be related to the experience of using these vaccines under field condition. Considering that both $\mathrm{HN}$ and $\mathrm{F}$ proteins induce the production of antibodies involved in NDV neutralization (Merz et al. 1980; Boursnell et al. 1990; Naohiro et al. 1994; Reynolds and Maraqa 2000), they were used for antibody epitope finding.

Live vaccines are the most commonly used vaccine type for immunization of chickens against NDV (Dimitrov et al. 2017). The LaSota, B1, and VG/GA vaccine strains belong to genotype II within class II. Among these strains, LaSota has the highest replicative capacity in chickens, VG/GA is enterotropic and B1 is the most attenuated vaccine strain (Dimitrov et al. 2017). I-2, V4, Ulster, and PHY-LMV42 vaccine strains belong to genotype I within class II and have been used for chickens of all ages due to their high safety profile (Dimitrov et al. 2017). V4 and I-2 vaccine strains are thermostable and are especially suitable for use in rural areas. The activity of these two strains is maintained in freeze-dry form for up to two months at temperatures between 9 and $29^{\circ} \mathrm{C}$ and up to two weeks at temperatures between 30 and $37^{\circ} \mathrm{C}$ (Alders 2014). R2B and $\mathrm{F}$ vaccine strains, belong to genotype II within class II, are respectively mesogenic and lentogenic (Chellappa et al. 2012; Dey et al. 2014). R2B strain can be used in adult chickens previously immunized with a lentogenic vaccine and strain $\mathrm{F}$ can be used in young chickens (Chellappa et al. 2012; Dey et al. 2014).

All genotypes of NDV belong to a single serotype (Dimitrov et al. 2019), and the use of classical vaccine strains such as LaSota, B1, Ulster, and VG/GA has provided complete protection against clinical signs in chickens. However, under field condition, these vaccines have not been able to completely prevent infection, virus shedding, and thus the spread of a virulent NDV; however, the use of vaccines with antigen similarity to the field strain has been reported effective in eliminating these disadvantages (Miller et al. 2007; Jeon et al. 2008; Miller et al. 2009; Miller et al. 2013; Liu et al. 2017). Opposed to these findings, Dortmans et al. found that the lower efficacy of classical vaccine strains attributed to inadequate vaccination practices but not antigenic variation (Dortmans et al. 2012).

In this study, different parameters including the evolutionary distance, identity, and linear and conformational antibody epitopes of $\mathrm{F}$ and $\mathrm{HN}$ proteins of a sub-genotype VII.2 NDV and nine vaccine strains were evaluated. Estimates of evolutionary distance showed that the number of amino acid substitutions per site between sequences is higher for $\mathrm{HN}$ compared to $\mathrm{F}$ protein (Table 1 ). This is consistent with the notion that $\mathrm{HN}$ protein is highly under immunological pressure and changes more rapidly (Gong and Cui 2011; Gu et al. 2011).

To increase the accuracy of B-cell linear epitopes prediction and the need for antigen 3D structure information to predict B-cell conformational epitopes, molecular modeling of $\mathrm{F}$ and $\mathrm{HN}$ proteins was performed for the studied viruses. Since the quality of the predicted 3D structures is influential in the 
epitope finding, the accuracy of 3D structure prediction was investigated and confirmed in different ways (Table 2). Observing the position of the predicted epitopes on the 3D structure of $\mathrm{F}$ and $\mathrm{HN}$ antigens (Figures 1 and 2) and comparing them with the experimentally detected neutralizing epitopes (Chambers et al. 1988; Toyoda et al. 1988; Neyt et al. 1989; Yusoff et al. 1989; lorio et al. 1991; lorio et al. 1992), confirmed the epitope mapping accuracy.

Since changes in amino acids may affect an antigen's 3D structure and conformational epitopes, analogous epitopes with different compositions and numbers of amino acids were observed (Tables 3-6). Studies have shown that even a single amino acid difference can inhibit the function of a monoclonal antibody in NDV neutralization (Chambers et al. 1988; Toyoda et al. 1988; Neyt et al. 1989; Yusoff et al. 1989; lorio et al. 1991; lorio et al. 1992). Due to the unknown role of each amino acid in binding to antibodies, vaccine strain epitopes with 100\% identity to their analogous epitopes of the VII.2 NDV were employed to find the most similar vaccine strain (Table 7). The range of identical epitopes of the vaccine strains to the VII.2 NDV was wider for F (3 to 10 epitopes) than HN protein (2 to 5 epitopes) (Table 7); contrary to this, the range of difference of evolutionary distance and protein sequence identity for $F$ and HN proteins were close to each other (Table 1). For some vaccine strains including B1, LaSota, Ulster, and PHY.LMV.42, the F protein was close to HN protein in the number of identical epitopes. However, for vaccine strains including $\mathrm{V} 4$ and $F$, the number of $F$ protein identical epitopes was more than two-fold higher compared to the HN protein (Table 7).

Although anti-HN antibodies can neutralize a paramyxovirus at the host attachment step, in the case of viruses that have entered and multiplied in the cell, anti-F antibodies can prevent the virus spread to nearby cells (Merz et al. 1980). Accordingly, an effective vaccine for paramyxoviruses should also be able to induce anti-F protein neutralizing antibodies. Based on this fact, it can be speculated that the wider range of differences in the number of identical epitopes in relation to $\mathrm{F}$ compared to $\mathrm{HN}$ protein may distinguish the efficacy of these vaccines not in the early stages of infection but in preventing virus cellto-cell transmission and the spread of infection (Table 7). In a study by Kim et al., it was found that $F$ protein plays the most important role in providing protection following the use of genotype-matched vaccines (Kim et al. 2013).

As the number of common epitopes between a vaccine strain and a virulent field NDV increases, the protective antibodies may be expanded (Miller et al. 2009; Miller et al. 2013; Yang et al. 2017; Shahar et al. 2018). This study showed that the V4 and I-2 strains have the most epitope similarity to the VII.2 NDV (Table 7). Among the vaccine strains, B1, PHY.LMV.42 and LaSota had the lowest number of identical epitopes to the VII.2 NDV.

However, the successful experience of using the LaSota vaccine compared to other classical vaccines such as V4 and I-2 may indicate that, especially in relation to the use of NDV live vaccines, epitope similarity is not the dominant factor in vaccine efficacy but higher replicative capacity and tropism of the LaSota vaccine plays a more important role in inducing protective responses. 


\section{Declarations}

Funding No funding was received for conducting this study.

Conflicts of interest/Competing interests The author has no relevant financial or non-financial interests to disclose.

Availability of data and material All data generated or analysed during this study are included in this published article [and its supplementary information files].

Code availability Not applicable

Authors' contributions Not applicable

Ethics approval Not applicable

Consent to participate Not applicable

Consent for publication Not applicable

\section{References}

Ababneh M, Ferreira HL, Khalifeh M, Suarez DL, Afonso CL, (2018). First genome sequence of Newcastle disease virus of genotype Vlli from Jordan. Microbiology Resource Announcements 7.

Abolnik C, Mubamba C, Wandrag DB, Horner R, Gummow B, Dautu G, Bisschop SP, (2018). Tracing the origins of genotype VII h Newcastle disease in Southern Africa. Transboundary and emerging diseases 65, e393-e403.

Alders RG, (2014). Making Newcastle disease vaccines available at village level. Veterinary Record 174, 502-503.

Barlow DJ, Edwards MS, Thornton JM, (1986). Continuous and discontinuous protein antigenic determinants. Nature 322, 747-748.

Boursnell M, Green P, Samson A, Campbell J, Deuter A, Peters R, Millar N, Emmerson P, Binns M, (1990). A recombinant fowlpox virus expressing the hemagglutinin-neuraminidase gene of Newcastle disease virus (NDV) protects chickens against challenge NDV. Virology 178, 297-300.

Chambers P, Nesbit M, Yusoff K, Millar N, Samson A, Emmerson P, (1988). Location of a neutralizing epitope for the haemagglutinin-neuraminidase glycoprotein of Newcastle disease virus. Journal of general virology $69,2115-2122$.

Chellappa MM, Dey S, Gaikwad S, Kataria J, Vakharia V, 2012. Complete genome sequence of Newcastle disease virus mesogenic vaccine strain R2B from India. Am Soc Microbiol. 
Chen L, Gorman JJ, McKimm-Breschkin J, Lawrence LJ, Tulloch PA, Smith BJ, Colman PM, Lawrence MC, (2001). The structure of the fusion glycoprotein of Newcastle disease virus suggests a novel paradigm for the molecular mechanism of membrane fusion. Structure 9, 255-266.

Crennell S, Takimoto T, Portner A, Taylor G, (2000). Crystal structure of the multifunctional paramyxovirus hemagglutinin-neuraminidase. Nature structural biology 7, 1068-1074.

Dey S, Chellappa MM, Gaikwad S, Kataria JM, Vakharia VN, (2014). Genotype characterization of commonly used Newcastle disease virus vaccine strains of India. PLoS One 9, e98869.

Dimitrov KM, Abolnik C, Afonso CL, Albina E, Bahl J, Berg M, Briand F-X, Brown IH, Choi K-S, Chvala I, (2019). Updated unified phylogenetic classification system and revised nomenclature for Newcastle disease virus. Infection, Genetics and Evolution 74, 103917.

Dimitrov KM, Afonso CL, Yu Q, Miller PJ, (2017). Newcastle disease vaccines-A solved problem or a continuous challenge? Veterinary microbiology 206, 126-136.

Dortmans JC, Peeters BP, Koch G, (2012). Newcastle disease virus outbreaks: vaccine mismatch or inadequate application? Veterinary microbiology 160, 17-22.

Fuller C, Löndt B, Dimitrov K, Lewis N, van Boheemen S, Fouchier R, Coven F, Goujgoulova G, Haddas R, Brown I, (2017). An epizootiological report of the re-emergence and spread of a lineage of virulent Newcastle disease virus into Eastern Europe. Transboundary and emerging diseases 64, 1001-1007.

Ghalyanchilangeroudi A, Hosseini H, Jabbarifakhr M, Fallah Mehrabadi MH, Najafi H, Ghafouri SA, Mousavi FS, Ziafati Z, Modiri A, (2018). Emergence of a virulent genotype Vlli of Newcastle disease virus in Iran. Avian Pathology 47, 509-519.

Gong Y, Cui Z, (2011). Epitope variation in the Newcastle disease virus HN gene under antibody immune selective pressure in cell culture. Science China Life Sciences 54, 474-479.

Gorman J, Nestorowicz A, Mitchell SJ, Corino GL, Selleck PW, (1988). Characterization of the sites of proteolytic activation of Newcastle disease virus membrane glycoprotein precursors. Journal of Biological Chemistry 263, 12522-12531.

Gu M, Liu W, Xu L, Cao Y, Yao C, Hu S, Liu X, (2011). Positive selection in the hemagglutininneuraminidase gene of Newcastle disease virus and its effect on vaccine efficacy. Virology journal 8, 150.

Heo L, Park H, Seok C, (2013). GalaxyRefine: Protein structure refinement driven by side-chain repacking. Nucleic Acids Res 41, W384-388.

ICTV, (2019). International committee on taxonomy of viruses. Virus Taxonomy. 
Iorio RM, Glickman RL, Sheehan JP, (1992). Inhibition of fusion by neutralizing monoclonal antibodies to the haemagglutinin-neuraminidase glycoprotein of Newcastle disease virus. Journal of general virology 73, 1167-1176.

Iorio RM, Syddall RJ, Sheehan JP, Bratt MA, Glickman RL, Riel AM, (1991). Neutralization map of the hemagglutinin-neuraminidase glycoprotein of Newcastle disease virus: domains recognized by monoclonal antibodies that prevent receptor recognition. Journal of virology $65,4999-5006$.

Jeon W-J, Lee E-K, Lee Y-J, Jeong O-M, Kim Y-J, Kwon J-H, Choi K-S, (2008). Protective efficacy of commercial inactivated Newcastle disease virus vaccines in chickens against a recent Korean epizootic strain. Journal of veterinary science $9,295-300$.

Khattar SK, Yan Y, Panda A, Collins PL, Samal SK, (2009). A Y526Q mutation in the Newcastle disease virus $\mathrm{HN}$ protein reduces its functional activities and attenuates virus replication and pathogenicity. Journal of virology $83,7779-7782$.

Kim S-H, Wanasen N, Paldurai A, Xiao S, Collins PL, Samal SK, (2013). Newcastle disease virus fusion protein is the major contributor to protective immunity of genotype-matched vaccine. PloS one 8, e74022.

Kumar S, Stecher G, Li M, Knyaz C, Tamura K, (2018). MEGA X: molecular evolutionary genetics analysis across computing platforms. Molecular biology and evolution 35, 1547-1549.

Lee Y-J, Sung H-W, Choi J-G, Lee E-K, Yoon H, Kim J-H, Song C-S, (2008). Protection of chickens from Newcastle disease with a recombinant baculovirus subunit vaccine expressing the fusion and hemagglutinin-neuraminidase proteins. Journal of Veterinary Science 9, 301-308.

Liu J, Zhu J, Xu H, Li J, Hu Z, Hu S, Wang X, Liu X, (2017). Effects of the HN antigenic difference between the vaccine strain and the challenge strain of Newcastle Disease virus on virus shedding and transmission. Viruses 9, 225.

Merz DC, Scheid A, Choppin P, (1980). Importance of antibodies to the fusion glycoprotein of paramyxoviruses in the prevention of spread of infection. The Journal of experimental medicine 151, 275288.

Miller PJ, Afonso CL, El Attrache J, Dorsey KM, Courtney SC, Guo Z, Kapczynski DR, (2013). Effects of Newcastle disease virus vaccine antibodies on the shedding and transmission of challenge viruses. Developmental \& Comparative Immunology 41, 505-513.

Miller PJ, Estevez C, Yu Q, Suarez DL, King DJ, (2009). Comparison of viral shedding following vaccination with inactivated and live Newcastle disease vaccines formulated with wild-type and recombinant viruses. Avian diseases 53, 39-49.

Miller PJ, King DJ, Afonso CL, Suarez DL, (2007). Antigenic differences among Newcastle disease virus strains of different genotypes used in vaccine formulation affect viral shedding after a virulent challenge. 
Vaccine $25,7238-7246$.

Moult J, Fidelis K, Kryshtafovych A, Schwede T, Tramontano A, (2018). Critical assessment of methods of protein structure prediction (CASP)-Round XII. Proteins: Structure, Function, and Bioinformatics 86, 7-15.

Naohiro K, Masahiro N, Mitsuru O, Chieko K, Yoshiharu M, Takeshi M, (1994). Protective effect of individual glycoproteins of Newcastle disease virus expressed in insect cells: the fusion protein derived from an avirulent strain had lower protective efficacy. Virus research 32, 373-379.

Neyt C, Geliebter J, Slaoui M, Morales D, Meulemans G, Burny A, (1989). Mutations located on both F1 and F2 subunits of the Newcastle disease virus fusion protein confer resistance to neutralization with monoclonal antibodies. Journal of virology 63, 952-954.

Ponomarenko J, Bui HH, Li W, Fusseder N, Bourne PE, Sette A, Peters B, (2008). ElliPro: a new structurebased tool for the prediction of antibody epitopes. BMC Bioinformatics 9, 514.

Reynolds D, Maraqa A, (2000). Protective immunity against Newcastle disease: the role of antibodies specific to Newcastle disease virus polypeptides. Avian diseases, 138-144.

Römer-Oberdörfer A, Veits J, Werner O, Mettenleiter TC, (2006). Enhancement of pathogenicity of Newcastle disease virus by alteration of specific amino acid residues in the surface glycoproteins $\mathrm{F}$ and HN. Avian diseases 50, 259-263.

Römer-Oberdörfer A, Werner O, Veits J, Mebatsion T, Mettenleiter TC, (2003). Contribution of the length of the $\mathrm{HN}$ protein and the sequence of the $\mathrm{F}$ protein cleavage site to Newcastle disease virus pathogenicity. Journal of General Virology 84, 3121-3129.

Roy A, Kucukural A, Zhang Y, (2010). I-TASSER: a unified platform for automated protein structure and function prediction. Nat Protoc 5, 725-738.

Sali A, Blundell TL, (1993). Comparative protein modelling by satisfaction of spatial restraints. J Mol Biol $234,779-815$.

Shahar E, Haddas R, Goldenberg D, Lublin A, Bloch I, Bachner Hinenzon N, Pitcovski J, (2018). Newcastle disease virus: is an updated attenuated vaccine needed? Avian Pathology 47, 467-478.

Suarez DL, Miller PJ, Koch G, Mundt E, Rautenschlein S, (2020). Newcastle disease, other avian paramyxoviruses, and avian metapneumovirus infections. Diseases of poultry, 109-166.

Toyoda T, Gotoh B, Sakaguchi T, Kida H, Nagai Y, (1988). Identification of amino acids relevant to three antigenic determinants on the fusion protein of Newcastle disease virus that are involved in fusion inhibition and neutralization. Journal of virology 62, 4427-4430. 
Van Regenmortel MHV, (1996). Mapping Epitope Structure and Activity: From One-Dimensional Prediction to Four-Dimensional Description of Antigenic Specificity. Methods 9, 465-472.

Wiederstein M, Sippl MJ, (2007). ProSA-web: interactive web service for the recognition of errors in threedimensional structures of proteins. Nucleic Acids Res 35, W407-410.

Yang H-m, Zhao J, Xue J, Yang Y-I, Zhang G-Z, (2017). Antigenic variation of LaSota and genotype VII Newcastle disease virus (NDV) and their efficacy against challenge with velogenic NDV. Vaccine 35, 2732.

Yusoff K, Nesbit M, McCartney H, Meulemans G, Alexander D, Collins M, Emmerson P, Samson A, (1989). Location of neutralizing epitopes on the fusion protein of Newcastle disease virus strain Beaudette $\mathrm{C}$. Journal of general virology $70,3105-3109$.

\section{Tables}

Tables 1-7 are available in the Supplementary Files.

\section{Figures}


1

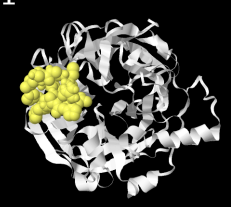

6

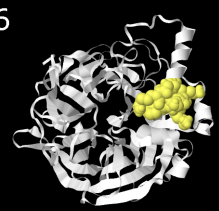

2
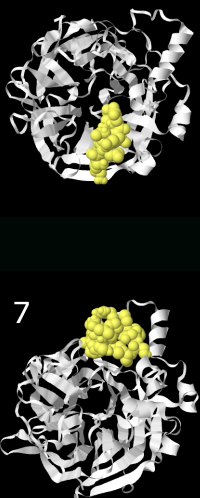

3

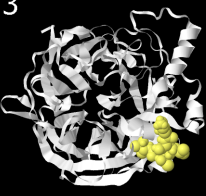

8

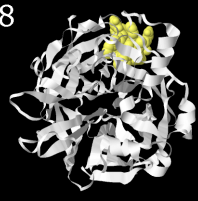

4
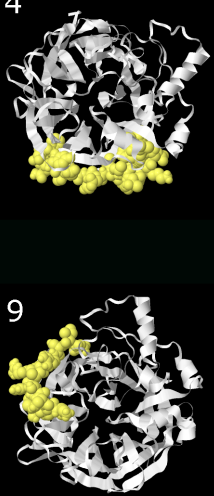
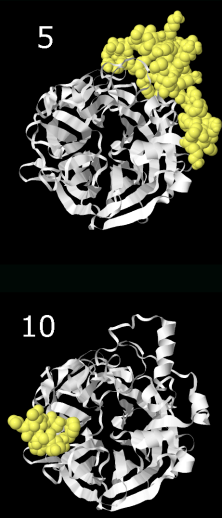

B

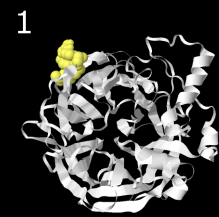

7

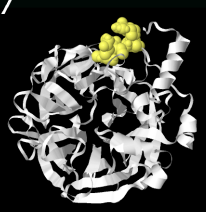

2

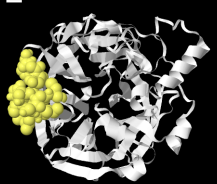

8

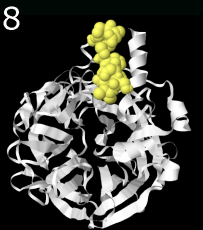

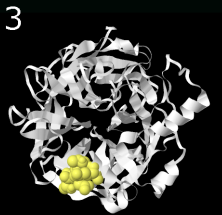

9

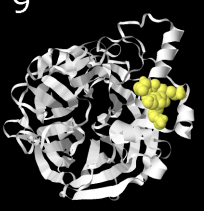

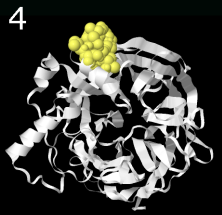
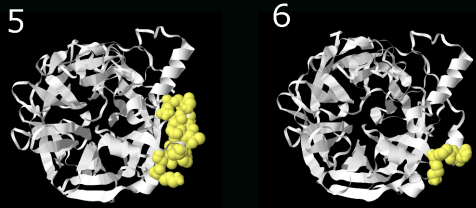

10
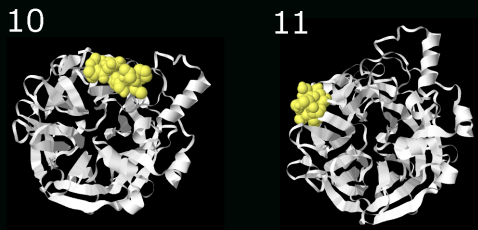

Figure 1

3D representation of linear and conformational B-cell epitopes for the HN protein of the VII.2 NDV. Panels $A$ and $B$ are showing the linear and conformational epitopes of HN protein, respectively. The number of each 3D structure corresponds to the number of epitopes listed in tables 3 and 4 . HN monomer ectodomain is shown in a way that the active site pocket is at the center and the amino acids with a potential role in inducing neutralizing epitopes are located around the active site pocket 

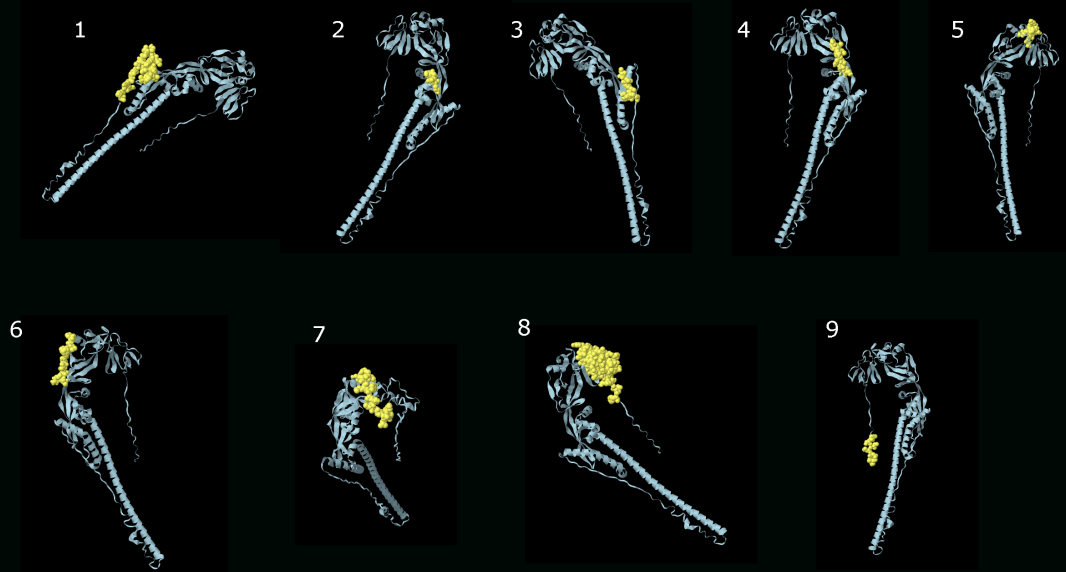

B
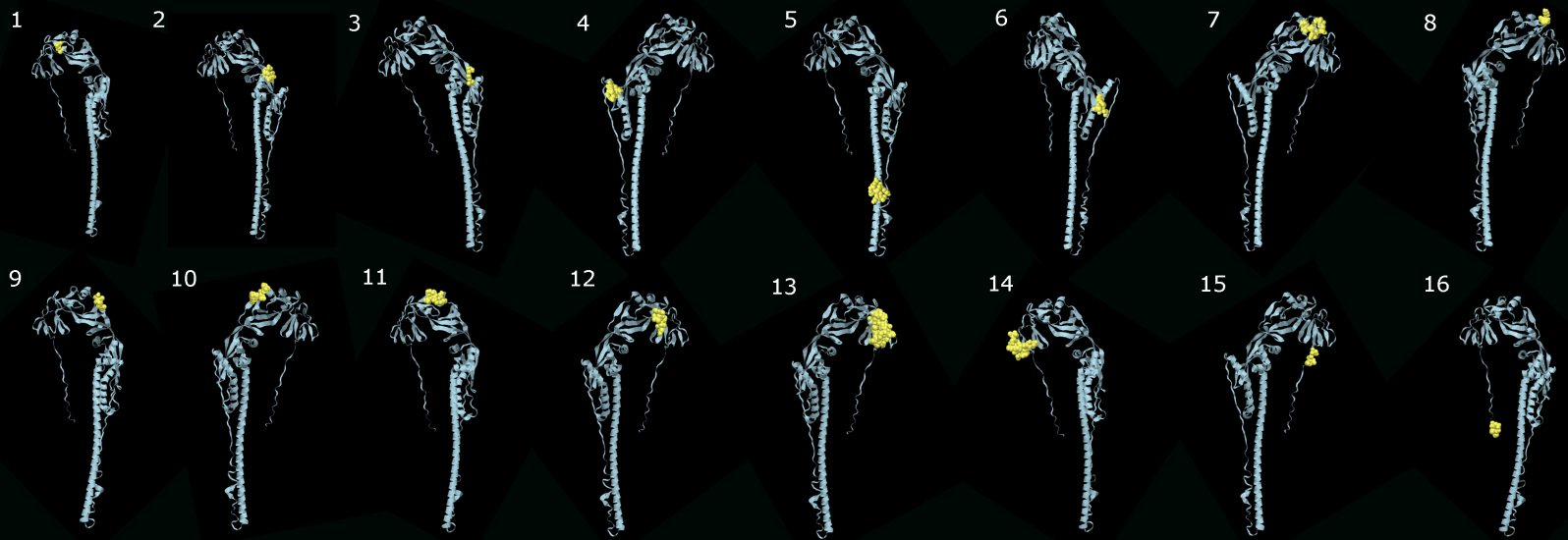

16

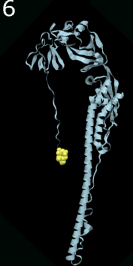

Figure 2

3D representation of predicted linear and conformational B-cell epitopes for F protein of the VII.2 NDV. Panels $A$ and $B$ are showing the linear and conformational epitopes of $F$ protein, respectively. The number of each 3D structure corresponds to the number of epitopes listed in tables 5 and 6

\section{Supplementary Files}

This is a list of supplementary files associated with this preprint. Click to download.

- Table1.docx

- Table2.docx

- Table3.docx 
- Table4.docx

- Table5.docx

- Table6.docx

- Table7.docx 